\title{
Novel Results on Slow Coherency in Consensus and Power Networks
}

\author{
Diego Romeres
}

\author{
Florian Dörfler
}

Francesco Bullo

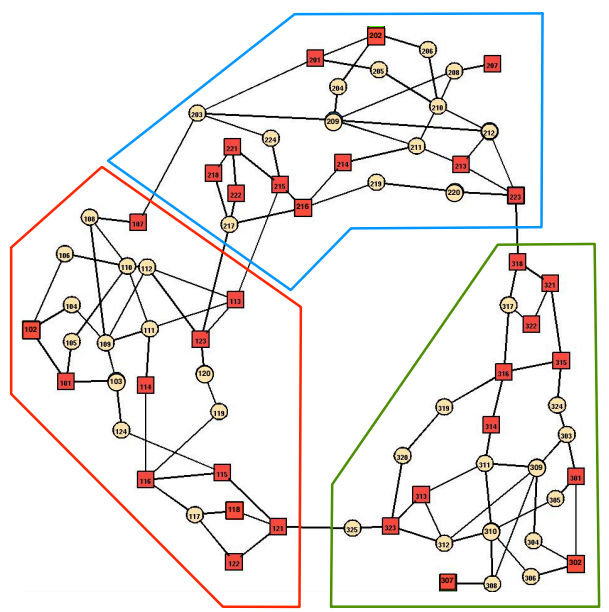

\begin{abstract}
We revisit the classic slow coherency and area aggregation approach to model reduction in power networks. The slow coherency approach is based on identifying sparsely and densely connected areas of a network, within which all generators swing coherently. A time-scale separation and singular perturbation analysis then results in a reduced loworder system, where coherent areas are collapsed into aggregate variables. Here, we study the application of slow coherency and area aggregation to first-order consensus systems and second-order power system swing dynamics. We unify different theoretic approaches and ideas found throughout the literature, we relax some technical assumptions, and we extend existing results. In particular, we provide a complete analysis of the second-order swing dynamics - without restrictive assumptions on the system damping. Moreover, we identify the reduced aggregate models as generalized first or second-order Laplacian flows with multiple time constants, aggregate damping and inertia matrices, and possibly adverse interactions.
\end{abstract}

\section{INTRODUCTION}

A power network is a large-scale and complex dynamical system. Here the attribute "complex" refers to both rich dynamics of the individual system component as well as their non-trivial interaction through the network. In order to tackle this complexity for analysis, control design, and monitoring schemes, it is of interest to construct reducedorder models which preserve the dynamics of interest. In this paper, we are interested in electromechanical inter-area dynamics, which are associated with the dynamics of power transfers and involve groups of generators oscillating relative to each other. In a heavily stressed grid, poorly damped interarea oscillations can even become unstable, see the blackout of August 10, 1996 in the Western American network [1].

To arrive at a better understanding of the complex interarea dynamics of a large-scale power grid, a natural approach is to collapse groups of coherent machines into single equivalent machines and study the dynamics of such a reduced model. Intuitively, these coherent groups can be identified as strongly connected components of the weighted graph, and entire geographic areas of a power grid can be aggregated to single equivalent models. The approach outlined above has been made precise in the pioneering work on slow coherency by Chow et al., see the seminal papers [2]-[6] among others. Slow coherency theory considers power network models, such as the RTS 96 in Figure 1, that are naturally partitioned into areas, which are internally densely connected and weakly connected among another. Next, aggregate variables

This material is based in part upon work supported by NSF grants IIS0904501 and CPS-1135819.

Diego Romeres, Florian Dörfler, and Francesco Bullo are with the Center for Control, Dynamical Systems and Computation, University of California at Santa Barbara. Email: diego.romeres@gmail.com and \{dorfler, bullo\}@engineering.ucsb.edu
Fig. 1. Illustration of RTS 96 power network with three areas. Here the square nodes are the generators and the circles the load buses of the network.

are defined for each area corresponding to the area's center of mass (or inertia). These aggregate variables describe the collective area dynamics, and they are complemented by a set of local variables representing the incoherency within the areas. A singular perturbation analysis shows that the long-term inter-area dynamics are determined by the aggregate variables. A recent application of slow coherency theory is measurement-based identification of the aggregate models for monitoring and control purposes [7].

In [2] and [3] the foundations of area aggregation are laid out for first and second-order linear interconnected systems with diffusive coupling. These networked systems correspond to either consensus dynamics [8], [9] or the electromechanical swing dynamics of network-reduced power systems [10], [11]. Recently, the methods developed in [2], [3] have been extended to non-linearly coupled first-order systems [12]. The second-order case has been heavily investigated in the context of power systems [2]-[6]. In [4] a sparsitybased technique is developed to identify coherent areas, and in [5] the singular perturbation and aggregation presented in [3] has been further refined. The articles [3]-[5] do not take the system damping into account, which is a technically inconvenient obstacle in the aggregation analysis. In [6], a singular perturbation analysis of a second-order dissipative system carried out, but the resulting slow and fast systems are required to have high frequency oscillations. Thus, some strong structural conditions on the initial system have to be met. Finally, in [2] an approach to second-order dissipative systems is presented, which is based on a restrictive uniform damping assumption and on an incomplete analysis.

In this paper, we review the different existing approaches 
[2]-[6], [12] to slow coherency in a unified language. We analyze the two cases of first-order consensus dynamics and second-order swing dynamics in power networks. We combine and extend the existing analysis approaches, and we remove some technical assumptions commonly made in the literature, such as regularity assumptions and assumptions on the graph connectivity. We also formally extend the existing theory to weighted graphs. More importantly, we provide a complete and detailed analysis of area aggregation for the second-order case with damping. The resulting aggregate model equals the one proposed in [2], [6], but we do not assume restrictive structural constraints or uniform damping. Finally, motivated by remarks in [3], we identify the reduced aggregate models as generalized consensus systems [8], [13] with multiple time constants (in the first-order case), aggregate damping and inertia matrices (in the second-order case), and possibly adverse interactions among the aggregate nodes (corresponding to negative coupling weights). We illustrate our developments with a simulations of the RTS 96 system.

In the interest of space we omit the explicit proofs and calculations in this paper, and we refer the reader to [14].

This paper is organized as follows. Section II presents the problem setup and summarizes the area aggregation process for consensus systems. Section III identifies the aggregate model as a generalized Laplacian system. Section IV extends the results from the first-order consensus dynamics to the second-order swing equations. Section V illustrates our theoretical results with a simulation study. Finally, Section VI concludes the paper. The remainder of this section recalls some preliminaries and introduces some notation.

\section{A. Preliminaries and notation}

Vectors and matrices: Let $\mathbf{1}_{n}$ and $\mathbf{0}_{n}$ be the $n$-dimensional vectors of unit and zero entries, respectively. Let $I_{n} \in \mathbb{R}^{n \times n}$ be the $n$-dimensional identity matrix. For a symmetric matrix $A=A^{T} \in \mathbb{R}^{n \times n}$ we write $A \succ 0, A \succeq 0, A \prec 0$, and $A \preceq 0$ if $A$ is positive definite, positive semidefinite, negative definite, negative semidefinite, respectively. Given an array $\left\{x_{i}\right\}_{i \in\{1, \ldots, n\}}$, let $x \in \mathbb{R}^{n}$ be the associated vector, and let $\operatorname{diag}(x) \in \mathbb{R}^{n \times n}$ be the associated diagonal matrix. For a function $f: \mathbb{R}^{n} \rightarrow \mathbb{R}$ and a constant $M \in \mathbb{R}$, we use the notation $f(x) \in \mathcal{O}(M)$ if there exists $k>0$ and $x_{0} \in \mathbb{R}^{n}$ such that $|f(x)| \leq k \cdot|M|$ for all $\|x\|_{\infty}>\left\|x_{0}\right\|_{\infty}$.

Sets: Given a discrete set $\mathcal{X}$, denote its cardinality by $|\mathcal{X}|$.

Algebraic graph theory: Consider a connected, undirected, and weighted graph $G=(\mathcal{V}, \mathcal{E}, W)$, where $\mathcal{V}=\{1, \ldots, n\}$ is the set of nodes, $\mathcal{E} \subset \mathcal{V} \times \mathcal{V}$ is the set of undirected edges, and $W=W^{T} \in \mathbb{R}^{n \times n}$ is the adjacency matrix with entries $w_{i j}>0$ if $\{i, j\} \in \mathcal{E}$ and $w_{i j}=0$ otherwise. Throughout the paper, we implicitly assume that all nonzero edge weights are uniformly non-degenerate and bounded, that is, there are $\underline{w}, \bar{w} \in \mathbb{R}$ such that for all $\{i, j\} \in \mathcal{E}, 0<\underline{w} \leq w_{i j} \leq \bar{w}$.

The following graph matrices and their properties will be of interest to us [15]. The degree matrix $D \in \mathbb{R}^{n \times n}$ is the diagonal matrix with elements $d_{i i}=\sum_{j=1, j \neq i}^{n} w_{i j}$. The Laplacian matrix $L=L^{T} \in \mathbb{R}^{n \times n}$ is defined by $L=$ $D-W$, and it satisfies $L \succeq 0$ and $L \mathbf{1}_{n}=\mathbf{0}_{n}$. If a number $\ell \in\{1, \ldots,|\mathcal{E}|\}$ and an arbitrary direction is assigned to each edge $\{i, j\} \in \mathcal{E}$, the (oriented) incidence matrix $B \in \mathbb{R}^{n \times|\mathcal{E}|}$ is defined component-wise by $B_{k \ell}=1$ if node $k$ is the sink node of edge $\ell$ and by $B_{k \ell}=-1$ if node $k$ is the source node of edge $\ell$; all other elements are zero. For $x \in \mathbb{R}^{n}$, the vector $B^{T} x$ has components $x_{i}-x_{j}$ corresponding to the oriented edge from $j$ to $i$. If $\operatorname{diag}\left(\left\{w_{i j}\right\}_{\{i, j\} \in \mathcal{E}}\right)$ is the diagonal matrix of edge weights, then $L=B \operatorname{diag}\left(\left\{w_{i j}\right\}_{\{i, j\} \in \mathcal{E}}\right) B^{T}$. If the graph is connected, then $\operatorname{Ker}\left(B^{T}\right)=\operatorname{Ker}(L)=\operatorname{span}\left(\mathbf{1}_{n}\right)$ and all $n-1$ non-zero eigenvalues of $L$ are strictly positive.

\section{Aggregation And Slow Coherency}

In this section, we introduce the problem setup in slow coherency analysis and present a brief yet complete analysis of time-scale separation and area aggregation.

\section{A. Mathematical models and problem setup}

Consider a connected, undirected, and weighted graph $G=(\mathcal{V}, \mathcal{E}, W)$ with $n$ nodes, Laplacian matrix $L$, and incidence matrix $B$. Associated to this graph, we consider two different dynamical systems. The first system is the widely adopted consensus protocol [3], [12], [13]. Consider $n$ autonomous agents. Each agent $i \in\{1, \ldots, n\}$ is equipped with a local state variable $x_{i}$, and the agents exchange their states according to the first-order consensus dynamics

$$
\dot{x}=-L x .
$$

A spectral analysis [13] of the consensus dynamics (1) reveals that asymptotically all agents synchronize to a common consensus state, that is, $\lim _{t \rightarrow \infty} x_{i}(t)=\lim _{t \rightarrow \infty} x_{j}(t)=$ $x_{\infty} \in \mathbb{R}$ for all $i, j \in\{1, \ldots, n\}$. In particular, the asymptotic consensus state is given by $x_{\infty}=\sum_{i=1}^{n} x_{i}(0) / n$.

A natural extension of the first-order consensus protocol (1) to a second-order mechanical system can be achieved as follows. With each node $i \in\{1, \ldots, n\}$, we associate an inertia coefficient $M_{i}>0$ and a damping coefficient $D_{i}>0$. Let $M \in \mathbb{R}^{n \times n}$ and $D \in \mathbb{R}^{n \times n}$ be the diagonal matrices of inertia and damping coefficients, and consider the secondorder dissipative consensus dynamics

$$
M \ddot{x}=-D \dot{x}-L x .
$$

Our enabling application of interest for the second-order consensus dynamics (2) is given by the electromechanical swing dynamics of large-scale electric power networks [2][6], [10]. Here each node $i \in\{1, \ldots, n\}$ corresponds to a synchronous generator or a load (modeled as a synchronous motor) with inertia $M_{i}$ damping $D_{i}$, and rotor angle $x_{i}$, and $L$ is the incremental admittance matrix, arising from a Jacobian linearization and Kron reduction [11] of the nonlinear power network dynamics. For the remainder of the paper, we refer to system (2) simply as power network dynamics.

In the following, we will assume that the graph $G$ is partitioned in $r$ areas, that is, $\mathcal{V}=\bigcup_{\alpha=1}^{r} \mathcal{V}_{\alpha}$ with $\mathcal{V}_{\alpha}$ being the node set of area $\alpha$. We denote the number of nodes in area $\alpha$ by $m_{\alpha}=\left|\mathcal{V}_{\alpha}\right|$. The edge set of area $\alpha$ is given by $\mathcal{E}_{\alpha}=\mathcal{E} \bigcap\left\{\mathcal{V}_{\alpha} \times \mathcal{V}_{\alpha}\right\}$. We assume that the partition is such that each subgraph $\left(\mathcal{V}_{\alpha}, \mathcal{E}_{\alpha}\right)$ is connected for $\alpha \in\{1, \ldots, r\}$. Finally, we define the internal set edge 
$\mathcal{E}_{\text {int }}$ by $\mathcal{E}_{\text {int }}=\bigcup_{\alpha=1}^{r} \mathcal{E}_{\alpha}$ and the external edge set $\mathcal{E}_{\text {ext }}$ by $\mathcal{E}_{\text {ext }}=\mathcal{E} \backslash \mathcal{E}_{\text {int }}$. Notice that $\mathcal{E}=\mathcal{E}_{\text {int }} \cup \mathcal{E}_{\text {ext }}$.

Accordingly, define the external adjacency matrix $W^{E} \in$ $\mathbb{R}^{n \times n}$ with elements $w_{i j}^{E}=w_{i j}$ if $\{i, j\} \in \mathcal{E}_{\text {ext }}$ and the internal adjacency matrix by $W^{I}=W-W^{E} \in$ $\mathbb{R}^{n \times n}$. The associated degree matrices are $D^{E} \in \mathbb{R}^{n \times n}$ with $d_{i i}^{E}=\sum_{j=1, j \neq i}^{n} w_{i j}^{E}$ and $D^{I} \in \mathbb{R}^{n \times n}$ with elements $d_{i i}^{I}=\sum_{j=1, j \neq i}^{n} w_{i j}^{I}$. Finally, define the internal Laplacian $L^{I}=D^{I}-W^{I} \in \mathbb{R}^{n \times n}$ and the external Laplacian $L^{E}=D^{E}-W^{E} \in \mathbb{R}^{n \times n}$. By construction, we obtain

$$
L=L^{I}+L^{E} \text {. }
$$

Accordingly, define $B^{I}$ and $B^{E}$ as the incidence matrices $B$ associated to $L^{I}=B^{I} \operatorname{diag}\left(\left\{w_{i j}\right\}_{\{i, j\} \in \mathcal{E}_{\text {int }}}\right) B^{I}$ and $L^{E}=$

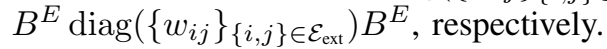

In the following, we will be particularly interested in the case, where each of the $r$ areas is internally densely connected, and distinct areas are sparsely connected among another. Given such a partition of the graph, it is reasonable to expect that nodes within each area strongly interact with each other and quickly synchronize their states $x_{i}(t)$ according to the inner-area dynamics induced by $L^{I}$. On the other hand, we also expect that nodes from disjoint areas interact only weakly, and the long-term behavior of (1) and (2) will depend mostly on inter-area dynamics induced by $L^{E}$ rather than inner-area dynamics. In the following subsections we make this intuition precise and formalize the particular notions of dense and sparse connections, time-scale separation, as well as inter-area and inner-area dynamics.

\section{B. Characterization of connectivity and sparsity}

We quantify the trade-off of internally densely connected and externally sparsely connected areas by two numerical parameters: a node parameter and an area parameter.

For each area $\alpha \in\{1, \ldots, r\}$ and each node $i \in \mathcal{V}_{\alpha}$, define the internal degree $c_{\alpha, i}^{I}$ and the external degree $c_{\alpha, i}^{E}$ by

$$
c_{\alpha, i}^{I}=\sum_{\{i, j\} \in \mathcal{E}_{\alpha}} w_{i j} \quad \text { and } \quad c_{\alpha, i}^{E}=\sum_{\{i, j\} \in \mathcal{E}_{\mathrm{ext}}} w_{i j} .
$$

The node parameter $d$ is then given by the worst-case ratio of the internal and external degree over all nodes and areas:

$$
d=\frac{\max _{\alpha \in\{1, \ldots, r\}, i \in \mathcal{V}_{\alpha}} c_{\alpha, i}^{E}}{\min _{\alpha \in\{1, \ldots, r\}, i \in \mathcal{V}_{\alpha}} c_{\alpha, i}^{I}}=\frac{c^{E}}{c^{I}} .
$$

For each area $\alpha \in\{1, \ldots, r\}$, define the internal edge weight $\gamma_{\alpha}^{I}$ and the external edge weight $\gamma_{\alpha}^{E}$ by

$$
\gamma_{\alpha}^{I}=\sum_{\{i, j\} \in \mathcal{E}_{\alpha}} w_{i j} \quad \text { and } \quad \gamma_{\alpha}^{E}=\sum_{\{i, j\} \in \mathcal{E}_{\mathrm{ext}} \cap i \in \mathcal{V}_{\alpha}} w_{i j} .
$$

Analogously to the node parameter, we aim to define an area parameter by the worst-case ratio of the internal and external edge weight. Notice that $\gamma_{\alpha}^{I} \geq \underline{m} c^{I}$, with $\underline{m}=\min _{\alpha}\left(m_{\alpha}\right)$, and $m_{\alpha}$ is the number of nodes in area $\alpha$.

We define the area parameter $\delta$ by

$$
\delta=\frac{\max _{\alpha \in\{1, \ldots, r\}} \gamma_{\alpha}^{E}}{\underline{m} c^{I}}=\frac{\gamma^{E}}{\underline{m} c^{I}}
$$

The two parameters $d$ and $\delta$ characterize and quantify the trade-off between connectivity inside the areas and among separate areas. Clearly, if $d \ll 1$, then each node has more internal than external weighted connections. Likewise, if $\delta \ll 1$ each area has more internal than external weighted connections. In this paper, we assume that the graph $G$ is partitioned such that $d \ll 1$ and $\delta \ll 1$, see for instance the RTS 96 power network example in Figure 1. We refer to [2], [4] for constructive algorithms to identify such a partitioning.

\section{Time-scale separation and singular perturbation analysis}

In the following, we will focus on the first-order consensus dynamics (1) and decompose them into fast local dynamics within each area and network-wide slow motions between the areas. We will postpone the analysis of the second-order power network dynamics (2) to Section IV.

To describe the slow inter-area motion, we define the slow aggregate variable $y_{\alpha} \in \mathbb{R}$ by the center of mass of area $\alpha$ :

$$
y_{\alpha}=\sum_{i \in \mathcal{V}_{\alpha}} \frac{x_{i}^{\alpha}}{m_{\alpha}}=\frac{1}{m_{\alpha}} \mathbf{1}_{m_{\alpha}}^{T} x^{\alpha}, \quad \alpha \in\{1, \ldots, r\}
$$

where $x_{i}^{\alpha}$ is the $i$ th component of $x$ for $i \in \mathcal{V}_{\alpha}$ and $x^{\alpha}=$ $\left[x_{1}^{\alpha}, \ldots, x_{m_{\alpha}}^{\alpha}\right]^{T}$. Accordingly, $y \in \mathbb{R}^{r}$ is the concatenated vector of slow aggregate variables defined by

$$
y=M_{a}^{-1} U^{T} x
$$

where $M_{a}=\operatorname{diag}\left(m_{1}, \ldots, m_{r}\right) \in \mathbb{R}^{r \times r}$ and $U$ is the blockdiagonal matrix $U=\operatorname{blkdiag}\left(\mathbf{1}_{m_{1}}, \ldots, \mathbf{1}_{m_{r}}\right) \in \mathbb{R}^{n \times r}$.

The fast inner-area motion is given by a weighted difference between the state of the nodes in each area, and different metrics have been proposed for this weighted difference [3], [12]. Here, we present the definition from [12] and define the fast local variable $z_{\alpha} \in \mathbb{R}^{m_{\alpha}-1}$ of area $\alpha$ as

$$
z_{\alpha}=Q_{\alpha} x^{\alpha}, \quad \alpha \in\{1, \ldots, r\},
$$

where the matrix $Q_{\alpha} \in \mathbb{R}^{m_{\alpha}-1 \times m_{\alpha}}$ is defined by

$$
Q_{\alpha}=\left[\begin{array}{ccccc}
-1+\left(m_{\alpha}-1\right) v & 1-v & -v & \ldots & -v \\
-1+\left(m_{\alpha}-1\right) v & -v & 1-v & \ldots & -v \\
\vdots & \vdots & \vdots & \ddots & \vdots \\
-1+\left(m_{\alpha}-1\right) v & -v & -v & \ldots & 1-v
\end{array}\right]
$$

with $v=\frac{m_{\alpha}-\sqrt{m_{\alpha}}}{m_{\alpha}\left(m_{\alpha}-1\right)}<1$. Accordingly, let $Q=$ $\operatorname{blkdiag}\left(Q_{1}, \ldots, Q_{r}\right) \in \mathbb{R}^{n-r \times n}$, and let $z \in \mathbb{R}^{n-r}$ be the vector of fast variables $z_{\alpha}$ defined by

$$
z=Q x
$$

Compared to other choices of fast variables [3], the construction in (8) features the following convenient properties.

Lemma 2.1: (Properties of $Q_{\alpha}$ ) Consider the matrix $Q_{\alpha}$ in (8). The matrix $Q_{\alpha}$ features zero row sums and orthonormal rows, that is, $Q_{\alpha} \mathbf{1}_{m_{\alpha}}=0$ and $Q_{\alpha} Q_{\alpha}^{T}=I_{m_{\alpha}-1}$.

From (7) and (9), we obtain the transformation of the original state $x$ into the aggregate and local variables:

$$
\left[\begin{array}{l}
y \\
z
\end{array}\right]=\left[\begin{array}{l}
C \\
Q
\end{array}\right] x
$$

where $C=M_{a}^{-1} U^{T}$. Due to Lemma 2.1, the inverse of the transformation (10) is explicitly given by

$$
x=\left[\begin{array}{ll}
U & Q^{T}
\end{array}\right]\left[\begin{array}{l}
y \\
z
\end{array}\right]
$$


By means of the coordinate transformation (10)-(11), the dynamics (1) read in local and aggregate variables as

$$
\left[\begin{array}{c}
\dot{y} \\
\dot{z}
\end{array}\right]=\left[\begin{array}{ll}
\bar{A}_{11} & \bar{A}_{12} \\
\bar{A}_{21} & \bar{A}_{22}
\end{array}\right]\left[\begin{array}{l}
y \\
z
\end{array}\right],
$$

where we used the fact that $L^{I} U=\mathbf{0}_{n}$ and the shorthands

$$
\begin{array}{ll}
\bar{A}_{11}=-C L^{E} U, & \bar{A}_{12}=-C L^{E} Q^{T}, \\
\bar{A}_{21}=-Q L^{E} U, & \bar{A}_{22}=-Q\left(L^{I}+L^{E}\right) Q^{T} .
\end{array}
$$

The submatrices in (13) are obtained using the Laplacian decomposition (3) and from the fact $C$ and $U$ span the left and right nullspace of $L^{I}$, respectively.

Lemma 2.2: (Order relations I) The $\infty$-norms (row sums) of the submatrices in (13) satisfy

$$
\begin{aligned}
& \left\|\bar{A}_{11}\right\|_{\infty}=\left\|C L^{E} U\right\|_{\infty} \in \mathcal{O}\left(c^{I} \delta\right), \\
& \left\|\bar{A}_{12}\right\|_{\infty}=\left\|C L^{E} Q^{T}\right\|_{\infty} \in \mathcal{O}\left(c^{I} \delta\right), \\
& \left\|\bar{A}_{21}\right\|_{\infty}=\left\|Q L^{E} U\right\|_{\infty} \in \mathcal{O}\left(c^{I} d\right), \\
& \left\|\bar{A}_{22}\right\|_{\infty}=\left\|Q\left(L^{I}+L^{E}\right) Q^{T}\right\|_{\infty} \in \mathcal{O}\left(c^{I}\right)
\end{aligned}
$$

In comparison with the corresponding result in [3], Lemma 2.2 provides an upper bound on $\left\|\bar{A}_{22}\right\|_{\infty}$ without additional assumptions, such as placing a lower bound on $\left|\mathcal{E}_{\alpha}\right|$.

Lemma 2.2 suggests a two time-scale separation of the transformed dynamics (12) into the fast time scale $t_{f}=c^{I} t$ and the slow time scale $t_{s}=\delta t_{f}$. Furthermore, Lemma 2.2 suggests a rescaling of the submatrices in (12) as follows:

$$
\begin{array}{ll}
A_{11}=\frac{\bar{A}_{11}}{c^{I} \delta}, & A_{12}=\frac{\bar{A}_{12}}{c^{I} \delta}, \\
A_{21}=\frac{\bar{A}_{21}}{c^{I} d}, & A_{22}=\frac{\bar{A}_{22}}{c^{I}} .
\end{array}
$$

Notice that all submatrices $A_{i j}$ are scale-free, that is, each $\left\|A_{i j}\right\|_{\infty} \in \mathcal{O}(1)$ for $i, j \in\{1,2\}$.

Lemma 2.3: (Regularity) The matrix $A_{22}$ is non-singular. In comparison to the analogous results in [3] and in [12], Lemma 2.3 shows the non singularity of $A_{22}$ without additional assumptions such as $d \ll 1$.

By rescaling the submatrices in (12) as in (14) and rescaling time as $t_{s}=\delta t_{f}$, the system (12) can be equivalently rewritten in singular perturbation standard form

$$
\begin{aligned}
\frac{d y}{d t_{s}} & =A_{11} y+A_{12} z, \\
\delta \frac{d z}{d t_{s}} & =d A_{21} y+A_{22} z .
\end{aligned}
$$

A standard singular perturbation analysis [16, Section 11] applied to (15) yields the slow reduced system (defined for the slow aggregate variable $y$ and in the scale $t_{s}$ ) as

$$
\frac{d y_{s}}{d t_{s}}=\left(A_{11}-d A_{12} A_{22}^{-1} A_{21}\right) y_{s}=A_{0} y_{s}, \quad y_{s}(0)=y(0) \text {. }
$$

The corresponding fast boundary layer system (defined for the fast local variable $z$ and in the scale $t_{f}$ ) is obtained as

$$
\frac{d z_{f}}{d t_{f}}=A_{22} z_{f}, \quad z_{f}(0)=z(0)+d A_{22}^{-1} A_{21} y(0) .
$$

Tikhononov's Theorem [16, Theorem 11.2] applied to the singularly perturbed system (15) then yields the following result whose proof can be found in [3, Theorem 3.1].

Theorem 2.4: (Singular perturbation approximation I) Consider the singularly perturbed system (15) with solution denoted by $\left(y\left(t_{s}\right), z\left(t_{s}\right)\right)$, the boundary layer system (17) with solution denoted by $z_{f}\left(t_{f}\right)$, as well as the slow reduced system (16) with solution $y_{s}\left(t_{s}\right)$. There exist $\delta^{*}, d^{*}>0$ such that for all $0<\delta \leq \delta^{*}, 0<d \leq d^{*}$ the slow and fast motions of (15) are (16) and (17), respectively, and their solutions approximate the solution of (15) as follows:

$$
\begin{aligned}
& y\left(t_{s}\right)=y_{s}\left(t_{s}\right)+\mathcal{O}(\delta d), \\
& z\left(t_{s}\right)=-d A_{22}^{-1} A_{21} y_{s}\left(t_{s}\right)+z_{f}\left(t_{f}\right)+\mathcal{O}(\delta d) .
\end{aligned}
$$

\section{Properties of the Aggregate Model}

In this section, we analyze the properties of the aggregate model (16), where each area is collapsed into a single aggregate node. The system matrix of the aggregate model (16) can be rewritten in an insightful way by defining

$$
\begin{aligned}
L_{a} & \triangleq U^{T} L^{E} U, \\
L_{a}^{I} & \triangleq U^{T} L^{E} Q^{T}\left(Q L Q^{T}\right)^{-1} Q L^{E} U, \\
L_{\mathrm{red}} & \triangleq\left(L_{a}-L_{a}^{I}\right) .
\end{aligned}
$$

Lemma 3.1: (Reformulation of the aggregate model I) The aggregate model (16) reads equivalently as

$$
M_{a} \frac{d y_{s}}{d t}=-L_{\text {red }} y_{s} .
$$

Notice that the aggregate model (20) is presented in time scale $t$. This formulation avoids the dependency on the parameter $\delta$, and it will illuminate the connections between the aggregate model (20) the original model (1). Our analysis of the aggregate model is organized as follows: first, we show that the matrices $L_{a}, L_{a}^{I}$ and $L_{\text {red }}$ share some similarities with Laplacian matrices. Second, we state a convergence result connecting the aggregate the original model.

In the following, we refer to a matrix $A \in \mathbb{R}^{n \times n}$ as a generalized Laplacian matrix if it is symmetric, positive semidefinite, it features a simple zero eigenvalue and it has zero row and column sums. Notice that a generalized Laplacian matrix necessarily has positive diagonal elements, but compared to a conventional Laplacian matrix it may have also positive off-diagonal elements corresponding to negatively weighted edges in the associated graph.

In the following, we term the matrix $L_{\mathrm{red}}=L_{a}-L_{a}^{I}$ in (20) as the reduced Laplacian matrix. This terminology is justified by the following results.

Theorem 3.2: (Laplacian properties of the aggregate model I) Consider the matrices $L_{a}, L_{a}^{I}$, and $L_{\text {red }}$ defined in (19), respectively. The following properties hold:

1) $L_{a}$ is a Laplacian matrix;

2) $L_{a}^{I}$ is a symmetric and positive semidefinite matrix with zero row and column sums; and

3) $L_{\text {red }}$ is a generalized Laplacian matrix.

Remark 1: (Graphs associated to the reduced Laplacian matrices) The reduced Laplacian is obtained as the difference of the Laplacians $L_{a}$ and $L_{a}^{I}$. The matrix $L_{a}$ is the 
Laplacian corresponding to the aggregate graph, where each area is collapsed into a single node. The matrix $L_{a}^{I}$ shows the contribution of the area-internal topology and weights to the reduced Laplacian $L_{\text {red }}$. Whereas $L_{a}$ is a Laplacian matrix with positive edge weights, the matrix $L_{a}^{I}$ itself as well as $L_{\text {red }}$ can possibly feature negative edge weights. Hence, the reduced system can possibly feature adverse interactions. $\square$

Corollary 3.3: (Consensus convergence) Consider the aggregate model (20) and the original consensus model (1). The following statements hold:

1) The aggregate model (20) synchronizes exponentially to

$$
y_{s \infty} \cdot \mathbf{1}_{r}=\frac{\sum_{\alpha=1}^{r} m_{\alpha} y_{\alpha}(0)}{\sum_{\alpha=1}^{r} m_{\alpha}} \cdot \mathbf{1}_{r} .
$$

2) The consensus model (1) synchronizes exponentially to

$$
x_{\infty} \cdot \mathbf{1}_{n}=\frac{\sum_{i=1}^{n} x_{i}(0)}{n} \cdot \mathbf{1}_{n} .
$$

Moreover, we have that $y_{s_{\infty}}=x_{\infty}$.

\section{Aggregation in Power Networks}

In the following, we extend the results derived in the Sections II and III from the first-order consensus system (1) to the second-order system (2), which models the electromechanical swing dynamics of an interconnected power grid.

Analogous to Subsection II-B, we define the quantities $d$ and $\delta$ as in (4) and (5). We deviate from the first-order model (1) by accounting for different generator inertia coefficients, and we define the slow aggregate variable by

$$
y=C_{a} x=M_{a}^{-1} U^{T} M x,
$$

where $M_{a} \triangleq U^{T} M U$ and $C_{a}=M_{a}^{-1} U^{T} M$. Thus, $y_{\alpha}$ corresponds to the center of inertia angle of the area $\alpha$. We maintain the fast local variable $z=Q x$ and obtain

$$
\left[\begin{array}{c}
y \\
z
\end{array}\right]=\left[\begin{array}{c}
C_{a} \\
Q
\end{array}\right] x \quad, \quad\left[\begin{array}{c}
\dot{y} \\
\dot{z}
\end{array}\right]=\left[\begin{array}{c}
C_{a} \\
Q
\end{array}\right] \dot{x} .
$$

The inverse coordinate transformation then reads as

$$
x=\left[\begin{array}{ll}
U & M^{-1} Q^{T}\left(Q M^{-1} Q^{T}\right)^{-1}
\end{array}\right]\left[\begin{array}{l}
y \\
z
\end{array}\right] .
$$

This inverse transformation is the extension of (11) accounting for non-identical inertia coefficients, and it has been presented in [5] with a different matrix $Q$. Accordingly, we also define the diagonal matrix $D_{a} \triangleq U^{T} D U \in \mathbb{R}^{r \times r}$ representing the aggregate damping of each area.

The power network dynamics (2) can then be equivalently reformulated in the fast and slow variables as

$$
\begin{gathered}
{\left[\begin{array}{c}
\ddot{y} \\
\ddot{z}
\end{array}\right]=-\left[\begin{array}{cc}
M_{a}^{-1} D_{a} & \mathbf{0}_{r \times(n-r)} \\
Q M^{-1} D U & Q M^{-1} D Q^{T}
\end{array}\right]\left[\begin{array}{l}
\dot{y} \\
\dot{z}
\end{array}\right]} \\
-\left[\begin{array}{cc}
M_{a}^{-1} L_{a} & M_{a}^{-1} U^{T} L^{E} M^{-1} Q^{T}\left(Q M^{-1} Q^{T}\right)^{-1} \\
Q M^{-1} L^{E} U & Q M^{-1} L M^{-1} Q^{T}\left(Q M^{-1} Q^{T}\right)^{-1}
\end{array}\right]\left[\begin{array}{c}
y \\
z
\end{array}\right] .
\end{gathered}
$$

The submatrices which multiply the vector $\left[y^{T}, z^{T}\right]^{T}$ in (21) have a similar structure as those in (13). Analogous to the matrix $Q$, we define $\tilde{Q} \triangleq\left(Q M^{-1} Q^{T}\right)^{-1} Q$ satisfying $Q M^{-1} \tilde{Q}^{T}=I_{n-r}$ and $\tilde{Q} U=0_{n-r \times r}$. Consider the matrices

$$
\begin{aligned}
& \tilde{A}_{11}=\frac{-M_{a}^{-1} L_{a}}{c^{I} \delta}, \quad \tilde{A}_{12}=\frac{-M_{a}^{-1} U^{T} L^{E} M^{-1} \tilde{Q}^{T}}{c^{I} \delta} \\
& \tilde{A}_{21}=\frac{-Q M^{-1} L^{E} U}{c^{I} d}, \quad \tilde{A}_{22}=\frac{-Q M^{-1} L M^{-1} \tilde{Q}^{T}}{c^{I}} .
\end{aligned}
$$

Lemma 4.1: (Order relations and regularity II) The $\infty$ norms (row sums) of the submatrices in (22) satisfy

$$
\begin{array}{lrl}
\left\|\tilde{A}_{11}\right\|_{\infty} \in \mathcal{O}\left(c^{I} \delta\right), & \left\|\tilde{A}_{12}\right\|_{\infty} \in \mathcal{O}\left(c^{I} \delta\right), \\
\left\|\tilde{A}_{21}\right\|_{\infty} \in \mathcal{O}\left(c^{I} d\right), & \left\|\tilde{A}_{22}\right\|_{\infty} \in \mathcal{O}\left(c^{I}\right) .
\end{array}
$$

Moreover, the matrix $\tilde{A}_{22}$ is non-singular.

Analogous to the first-order system (1), we apply a change of time scale to bring the model (21) to singular perturbation standard form. For the double integrator system (21), the time scales to describe the fast and the slow motion are

$$
t_{f}=c^{I} t \quad, \quad t_{s}=\sqrt{\delta} t_{f}=\sqrt{\delta} c^{I} t .
$$

Accordingly, we define $\left[\bar{y}^{T}, \dot{\bar{y}}^{T}\right]^{T}=\left[y^{T}, \dot{y}^{T} / \sqrt{\delta}\right]^{T}$.

Lemma 4.2: (Power network model in singular perturbation standard form) Consider the power network dynamics (2) rewritten as in (21), the matrices in (22) and the parameters $\delta$ and $d$ defined in (5) and in (4), respectively. System (21) then reads equivalently as

$\frac{d}{d t_{s}}\left[\begin{array}{c}\bar{y} \\ \dot{\bar{y}} \\ \sqrt{\delta} z \\ \sqrt{\delta} \dot{z}\end{array}\right]=\left[\begin{array}{cccc}0 & \frac{I_{r}}{c^{I}} & 0 & 0 \\ \tilde{A}_{11} & -\tilde{D}_{1} & \tilde{A}_{12} & 0 \\ 0 & 0 & 0 & \frac{I_{n-r}}{c^{I}} \\ d \tilde{A}_{21} & -\frac{\sqrt{\delta} Q M^{-1} D U}{c^{I}} & \tilde{A}_{22} & -\frac{Q M^{-1} D Q^{T}}{c^{I}}\end{array}\right]\left[\begin{array}{c}\bar{y} \\ \bar{y} \\ z \\ \dot{z}\end{array}\right]$,

where the submatrix $\tilde{D}_{1}=\frac{M_{a}^{-1} D_{a}}{c^{I} \sqrt{\delta}}$ converges to a bounded and positive definite diagonal matrix as $\delta \rightarrow 0$.

System (23) has the same structure as system (15), and a singular perturbation analysis yields the slow reduced system

$$
\begin{aligned}
& \frac{d}{d t_{s}}\left[\begin{array}{c}
\bar{y}_{s} \\
\dot{\bar{y}}_{s}
\end{array}\right]=\left(R_{11}-R_{12} R_{22}^{-1} R_{21}\right) s=\left[\begin{array}{cc}
0 & \frac{I_{r}}{c^{I}} \\
\tilde{A}_{0} & -\bar{D}_{1}
\end{array}\right]\left[\begin{array}{c}
\bar{y}_{s} \\
\dot{\bar{y}}_{s}
\end{array}\right], \\
& {\left[\bar{y}_{s}(0) \dot{\bar{y}}_{s}(0)\right]^{T}=[\bar{y}(0) \dot{\bar{y}}(0)]^{T}}
\end{aligned}
$$

where $\tilde{A}_{0}=\tilde{A}_{11}-d \tilde{A}_{12} \tilde{A}_{22}^{-1} \tilde{A}_{21}, \bar{D}_{1}=\lim _{\delta \rightarrow 0} \frac{M_{a}^{-1} D_{a}}{c^{I} \sqrt{\delta}}$ is a bounded and positive definite diagonal matrix, and

$$
\begin{array}{rlrl}
R_{11} & =\left[\begin{array}{cc}
0 & \frac{I_{r}}{c^{I}} \\
\tilde{A}_{11} & -\bar{D}_{1}
\end{array}\right], & R_{12}=\left[\begin{array}{cc}
0 & 0 \\
\tilde{A}_{12} & 0
\end{array}\right], \\
R_{21}=\left[\begin{array}{cc}
0 & 0 \\
d \tilde{A}_{21} & 0
\end{array}\right], & R_{22}=\left[\begin{array}{cc}
0 & \frac{I_{n-r}}{c^{I}} \\
\tilde{A}_{22} & -\frac{Q M^{-1} D Q^{T}}{c^{I}}
\end{array}\right] .
\end{array}
$$

The corresponding fast boundary layer system is obtained as

$$
\begin{aligned}
& \frac{d}{d t_{s}}\left[\begin{array}{c}
z_{f} \\
\dot{z}_{f}
\end{array}\right]=R_{22}\left[\begin{array}{c}
z_{f} \\
\dot{z}_{f}
\end{array}\right] \\
& {\left[\begin{array}{c}
z_{f}(0) \\
\dot{z}_{f}(0)
\end{array}\right]=\left[\begin{array}{c}
z(0) \\
\dot{z}(0)
\end{array}\right]+d R_{22}^{-1} R_{21}\left[\begin{array}{c}
\bar{y}(0), \\
\dot{\bar{y}}(0)
\end{array}\right] .}
\end{aligned}
$$

The analog of Theorem 2.4 is then as follows:

Theorem 4.3: (Singular perturbation approximation II) Consider the singularly perturbed system (23) with solution denoted by $\left(\bar{y}\left(t_{s}\right), \dot{\bar{y}}\left(t_{s}\right), z\left(t_{s}\right), \dot{z}\left(t_{s}\right)\right)$, the boundary layer 
system (25) with solution denoted by $z_{f}\left(t_{f}\right), \dot{z}_{f}\left(t_{f}\right)$, as well as the slow reduced system (24) with solution $\bar{y}_{s}\left(t_{s}\right), \dot{\bar{y}}_{s}\left(t_{s}\right)$.

There exist $\delta^{*}, d^{*}>0$ such that for all $0<\delta \leq \delta^{*}$, $0<d \leq d^{*}$ the slow and fast motions of (23) are (24) and (25), respectively, and their solutions approximate the solution of (23) as follows:

$$
\begin{aligned}
{\left[\begin{array}{c}
\bar{y}\left(t_{s}\right) \\
\dot{\bar{y}}\left(t_{s}\right)
\end{array}\right] } & =\left[\begin{array}{c}
\bar{y}_{s}\left(t_{s}\right) \\
\dot{\bar{y}}_{s}\left(t_{s}\right)
\end{array}\right]+\mathcal{O}(\sqrt{\delta} d) \\
{\left[\begin{array}{c}
z\left(t_{s}\right) \\
\dot{z}\left(t_{s}\right)
\end{array}\right] } & =d R_{22}^{-1} R_{21}\left[\begin{array}{l}
\bar{y}_{s}\left(t_{s}\right) \\
\dot{\bar{y}}_{s}\left(t_{s}\right)
\end{array}\right]+\left[\begin{array}{c}
z_{f}\left(t_{f}\right) \\
\dot{z}_{f}\left(t_{f}\right)
\end{array}\right]+\mathcal{O}(\sqrt{\delta} d), \\
& =\left[\begin{array}{cc}
-d \tilde{A}_{22}^{-1} \tilde{A}_{21} & 0 \\
0 & 0
\end{array}\right]\left[\begin{array}{c}
\bar{y}_{s} \\
\dot{\bar{y}}_{s}
\end{array}\right]+\left[\begin{array}{c}
z_{f}\left(t_{f}\right) \\
\dot{z}_{f}\left(t_{f}\right)
\end{array}\right]+\mathcal{O}(\sqrt{\delta} d) .
\end{aligned}
$$

The system matrix of the second-order aggregate model (24) can be rewritten in an insightful way analogously to (20).

Theorem 4.4: (Reformulation and Laplacian properties of the aggregate model II) Let $M_{a}=U^{T} M U$ and $D_{a}=$ $U^{T} M U$ be the aggregate inertia and damping matrices. Then the aggregate model (24) reads equivalently as

$$
M_{a} \ddot{y}=-D_{a} \dot{y}-\tilde{L}_{\mathrm{red}} y,
$$

where $\tilde{L}_{\text {red }}$ is a generalized Laplacian matrix.

\section{Simulation Results for RTS 96 Power System}

We validate the theoretical developments in this article with the RTS 96 power network model shown in Figure 1. The RTS 96 consists of $n=33$ generators obeying the swing dynamics (2), the algebraic load flow is absorbed into the network parameters through Kron reduction [11], and the initial angles and frequencies are chosen to be aligned within each area. For illustrative purposes, we slightly increased the nominal generator damping constants (to reduce large oscillation amplitudes resulting in cluttered plots) and weakened the inter-area line connections by a factor 0.5 in the linearized model (corresponding to a steady state with large inter-area power transfers) resulting in $\delta=0.3955$.

The detailed simulation results are reported in Figure 2 and Figure 3 . Despite the fact that $\delta$ is not infinitesimally small, it can be observed that generators within an area swing coherently, and the reduced model (24) accurately approximates the aggregate behavior of the original model (23).

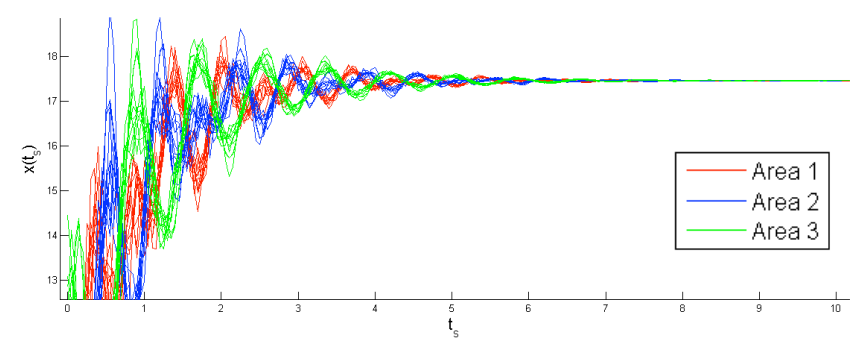

Fig. 2. Evolution of RTS 96 power network dynamics. All nodes within an area are plotted with the area color indicated in Figure 1.

\section{CONCLUSiOnS}

We studied area aggregation and model reduction of firstorder consensus and second-order power network dynamics based on slow coherency. We unified different solutions

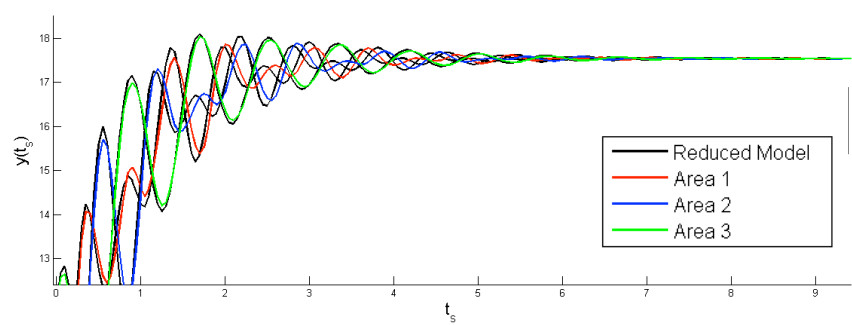

Fig. 3. Evolution of aggregate variable $y\left(t_{s}\right)$ in original model (23) of each area (plotted with the area color indicated in Figure 1) and the aggregate variable $\bar{y}\left(t_{s}\right)$ in the reduced model (24) (plotted in black).

found in the literature on slow coherency and area aggregation, we relaxed some technical assumptions, and we extended earlier results. Finally, we identified the corresponding reduced aggregate models as generalized Laplacian systems with multiple time constants, aggregate damping and inertia matrices, and possibly adverse interactions.

Our results point in an interesting future research direction, namely a graph-theoretic analysis relating the Laplacian of the original model and the generalized Laplacian of the reduced aggregate model. We are deeply convinced that a deeper understanding of the inter-area dynamics serves as a solid foundation for future control design.

\section{REFERENCES}

[1] V. Venkatasubramanian and Y. Li, "Analysis of 1996 Western American electric blackouts," in Bulk Power System Dynamics and ControlVI, Cortina d'Ampezzo, Italy, 2004.

[2] J. H. Chow, Time-scale modeling of dynamic networks with applications to power systems. Springer, 1982.

[3] J. H. Chow and P. Kokotović, "Time scale modeling of sparse dynamic networks," IEEE Transactions on Automatic Control, vol. 30, no. 8, pp. 714-722, 1985.

[4] J. H. Chow, J. Cullum, and R. A. Willoughby, "A sparsity-based technique for identifying slow-coherent areas in large power systems," IEEE Transactions on Power Apparatus and Systems, vol. 103, no. 3, pp. 463-473, 1984

[5] R. Date and J. Chow, "Aggregation properties of linearized two-timescale power networks," IEEE Transactions on Circuits and Systems, vol. 38, no. 7, pp. 720-730, 1991.

[6] J. H. Chow, J. J. Allemong, and P. V. Kokotović, "Singular perturbation analysis of systems with sustained high frequency oscillations," Automatica, vol. 14, no. 3, pp. 271-279, 1978.

[7] A. Chakrabortty, J. H. Chow, and A. Salazar, "A measurement-based framework for dynamic equivalencing of large power systems using wide-area phasor measurements," IEEE Transactions on Smart Grid, vol. 2, no. 1, pp. 56-69, 2011.

[8] F. Bullo, J. Cortés, and S. Martínez, Distributed Control of Robotic Networks. Princeton University Press, 2009.

[9] F. Garin and L. Schenato, "A survey on distributed estimation and control applications using linear consensus algorithms," in Networked Control Systems, ser. LNCIS, A. Bemporad, M. Heemels, and M. Johansson, Eds. Springer, 2010, pp. 75-107.

[10] P. W. Sauer and M. A. Pai, Power System Dynamics and Stability. Prentice Hall, 1998.

[11] F. Dörfler and F. Bullo, "Kron reduction of graphs with applications to electrical networks," IEEE Transactions on Circuits and Systems I: Regular Papers, vol. 60, no. 1, pp. 150-163, 2013.

[12] E. Biylk and M. Arcak, "Area aggregation and time-scale modeling for sparse nonlinear networks," Systems \& Control Letters, vol. 57, no. 2, pp. 142-149, 2007.

[13] R. Olfati-Saber, J. A. Fax, and R. M. Murray, "Consensus and cooperation in networked multi-agent systems," Proceedings of the IEEE, vol. 95, no. 1, pp. 215-233, 2007.

[14] D. Romeres, "Novel results on slow coherency in power networks," Master's thesis, Università di Padova, Italy, Dec. 2012.

[15] N. Biggs, Algebraic Graph Theory, 2nd ed. Cambridge University Press, 1994

[16] H. K. Khalil, Nonlinear Systems, 3rd ed. Prentice Hall, 2002. 